\title{
Erratum to: Head injuries and the risk of concurrent cervical spine fractures
}

\author{
Tuomo Thesleff $^{1}$ - Anneli Kataja ${ }^{2} \cdot$ Juha Öhman $^{1} \cdot$ Teemu M. Luoto $^{1}$
}

Published online: 23 March 2017

(C) Springer-Verlag Wien 2017

Erratum to: Acta Neurochir

DOI 10.1007/s00701-017-3133-0

Reason: Minor errors in Table 3 and related text "Patients with positive head CT scans $(2.2 \%, \mathrm{n}=6$, did not have more neurosurgical operations within 1 year post-HI due to cervical spine fractures compared with patients with negative head CT scans $(2.8 \%, \mathrm{n}=23 ; \mathrm{p}=0.614)$."

Corrected version: "Patients with positive head CT scans $(2.2 \%, \mathrm{n}=6)$ did not have more neurosurgical operations within one year post-HI due to CSIs compared with patients with negative head CT scans $(3.2 \%, \mathrm{n}=26 ; \mathrm{p}=0.431)$.”

The correct Table 3 is presented below:

The online version of the original article can be found at http://dx.doi. org/10.1007/s00701-017-3133-0

\footnotetext{
Tuomo Thesleff

tuomo.thesleff@pshp.fi

Anneli Kataja

anneli.kataja@pshp.fi

Juha Öhman

juha.ohman@pshp.fi

Teemu M. Luoto

teemu.luoto@pshp.fi

1 Department of Neuroscience and Rehabilitation, Tampere University Hospital, P.O. Box 2000, FI-33521 Tampere, Finland

2 Medical Imaging Centre, Department of Radiology, Tampere, University Hospital, P.O. Box 2000, FI-33521 Tampere, Finland
} 
Table 3 The CSI - characteristics of the study sample stratified into subgroups (head CT-positive patients, head CT-negative patients, and whole sample)

\begin{tabular}{|c|c|c|c|c|c|c|c|}
\hline Variable & $\begin{array}{l}\text { Head CT-positive } \\
\text { patients }(n=269)\end{array}$ & $\%$ & $\begin{array}{l}\text { Head CT-negative } \\
\text { patients }(n=822)\end{array}$ & $\%$ & $p$-value & $\begin{array}{l}\text { Whole sample } \\
(n=1091)\end{array}$ & $\%$ \\
\hline Cervical spine surgery within one year post injury & 6 & 2.2 & 26 & 3.2 & 0.431 & 32 & 2.9 \\
\hline Cervical spine fracture & 25 & 9.3 & 47 & 5.7 & 0.04 & 72 & 6.6 \\
\hline Cervical dislocation / subluxation & 8 & 3.0 & 23 & 2.8 & 0.880 & 31 & 2.8 \\
\hline Number of fractured cervical vertebrae & 35 & 13.0 & 66 & 8.0 & 0.014 & 101 & 9.3 \\
\hline $\mathrm{C} 0$ & 2 & 0.7 & 2 & 0.2 & 0.431 & 4 & 0.4 \\
\hline $\mathrm{C} 1$ & 5 & 1.9 & 5 & 0.6 & 0.062 & 10 & 0.9 \\
\hline C2 (odontoid) & 5 & 1.9 & 8 & 1.0 & 0.245 & 13 & 1.2 \\
\hline C2 (non-odontoid) & 5 & 1.9 & 11 & 1.3 & 0.538 & 16 & 1.5 \\
\hline $\mathrm{C} 3$ & 2 & 0.7 & 4 & 0.5 & 0.621 & 6 & 0.5 \\
\hline $\mathrm{C} 4$ & 1 & 0.4 & 8 & 1.0 & 0.344 & 9 & 0.8 \\
\hline $\mathrm{C} 5$ & 4 & 1.5 & 8 & 1.0 & 0.483 & 12 & 1.1 \\
\hline C6 & 8 & 3.0 & 9 & 1.1 & 0.031 & 17 & 1.6 \\
\hline $\mathrm{C} 7$ & 5 & 1.9 & 15 & 1.8 & 0.971 & 20 & 1.8 \\
\hline Multilevel cervical spine fracture (> 1 levels) & 9 & 3.3 & 13 & 1.6 & 0.074 & 22 & 2.0 \\
\hline Patients with lower level cervical fracture (C3-C7) & 16 & 5.9 & 31 & 3.8 & 0.127 & 47 & 4.3 \\
\hline Patients with upper level cervical fracture $(\mathrm{C} 0-\mathrm{C} 2)$ & 11 & 4.1 & 19 & 2.3 & 0.122 & 30 & 2.7 \\
\hline Spinal cord injury & 1 & 0.4 & 12 & 1.5 & & 13 & 1.2 \\
\hline ASIA-scale & & & & & 0.490 & & \\
\hline A & 1 & 0.4 & 2 & 0.2 & & 3 & 0.3 \\
\hline B & 0 & 0 & 4 & 0.5 & & 4 & 0.4 \\
\hline $\mathrm{C}$ & 0 & 0 & 5 & 0.6 & & 5 & 0.5 \\
\hline $\mathrm{D}$ & 0 & 0 & 1 & 0.1 & & 1 & 0.1 \\
\hline E or unknown & 268 & 99.6 & 810 & 98.5 & & 1078 & 98.8 \\
\hline
\end{tabular}

